\title{
Augmented circadian rhythm of the intrarenal renin-angiotensin systems in anti-thymocyte serum nephritis rats
}

\begin{abstract}
Shinsuke Isobe ${ }^{1}$, Naro Ohashi ${ }^{1}$, Sayaka Ishigaki ${ }^{1}$, Takayuki Tsuji ${ }^{1}$, Yukitoshi Sakao ${ }^{2}$, Akihiko Kato $^{2}$, Hiroaki Miyajima $^{1}$, Yoshihide Fujigaki ${ }^{1,3}$, Akira Nishiyama ${ }^{4}$ and Hideo Yasuda ${ }^{1}$

We report that disturbance to the circadian rhythm of urinary angiotensinogen (AGT) excretion may lead to renal damage, hypertension and diurnal blood pressure (BP) variations. We aim to clarify the circadian rhythm of the intrarenal renin-angiotensin system (RAS) and its contribution to renal damage, hypertension and BP variations, and to evaluate whether the administration of RAS blockers influences the circadian rhythms of intrarenal RAS components. Anti-thymocyte serum (ATS) nephritis rats were used as a chronic progressive glomerulonephritis model (group A) and compared with control rats (group C). Other rats with ATS nephritis received olmesartan medoxomil (an angiotensin II (AngII) type 1 receptor (AT1R) blocker; group $A O$ ) or hydralazine (a vasodilator; group AH). The levels of intrarenal RAS components were evaluated every $6 \mathrm{~h}$. The expression levels of intrarenal AGT, AngII and AT1R were increased in group A and peaked at the same time as BP and urinary protein excretion during the rest phase. The amplitude of the circadian fluctuation of these proteins was more increased in group $\mathrm{A}$ than in group $\mathrm{C}$. The circadian fluctuation of these proteins was reduced in groups $\mathrm{AO}$ and $\mathrm{AH}$. However, renal function, proteinuria and augmentation of intrarenal RAS components were reduced only in group AO. Intrarenal RAS components, such as AGT, Angll and AT1R proteins, were increased and the amplitude of the oscillations of these proteins was augmented in ATS nephritis rats. Interestingly, renal damage may be linked to the activation of the intrarenal RAS independent of the amplitude of its oscillations and BP.
\end{abstract}

Hypertension Research (2016) 39, 312-320; doi:10.1038/hr.2015.151; published online 7 January 2016

Keywords: anti-thymocyte serum nephritis; circadian rhythm; Intrarenal RAS; olmesartan medoxomil

\section{INTRODUCTION}

The circulating renin-angiotensin system (RAS) has a circadian rhythm ${ }^{1}$ and has an important role in blood pressure (BP) regulation. ${ }^{2}$ Although angiotensin II (AngII) is a strong vasoconstrictor, the circadian rhythm of circulating AngII levels is known to be independent of that of BP. ${ }^{3}$ It has been confirmed that activation of the intrarenal RAS has a critical role in the pathophysiology of hypertension and renal injury independent of the circulating RAS. ${ }^{2,4,5}$ Several studies have indicated that activation of the intrarenal RAS deteriorates the circadian rhythm of $\mathrm{BP}, 6,7$ and that the administration of an AngII type 1 receptor (AT1R) blocker (ARB) can ameliorate disturbed BP rhythm and intrarenal RAS activity in patients with hypertension or chronic kidney disease (CKD). ${ }^{7,8}$

Although it is clear that the circulating RAS has a circadian rhythm, reports of a circadian rhythm in the tissue-specific RAS are limited. Naito et al. demonstrated that cardiac RAS components have circadian rhythms, and that these oscillations are augmented under certain disease conditions. ${ }^{9}$ In this study, the administration of an ARB reduced the amplitude of oscillations of cardiac RAS components, although it is not known whether these effects were dependent on BP or RAS blockade. ${ }^{9}$ However, it is not clear whether the intrarenal RAS has a circadian rhythm or it influences renal damage and the circadian rhythm of BP. We previously reported that intrarenal RAS activity evaluated according to urinary angiotensinogen (AGT) levels was equally low during day and nighttime in individuals without CKD. In contrast, in patients with $\mathrm{CKD}$, the intrarenal RAS was activated and urinary AGT levels were higher at day than at nighttime. Moreover, urinary day and nighttime AGT levels were elevated to similar levels in CKD patients with the 'riser' BP pattern. In addition, urinary AGT levels were positively correlated with the levels of urinary protein and albumin excretion and BP. ${ }^{10}$ These results indicated that disturbance to the circadian rhythm of intrarenal RAS activation leads to renal damage and hypertension, which are both associated with diurnal BP variations. However, other RAS components besides AGT could not be evaluated, and the time course of urinary

${ }^{1}$ Internal Medicine 1, Hamamatsu University School of Medicine, Hamamatsu, Japan; ${ }^{2}$ Blood Purification Unit, Hamamatsu University School of Medicine, Hamamatsu, Japan;

${ }^{3}$ Department of Internal Medicine, Teikyo University School of Medicine, Tokyo, Japan and ${ }^{4}$ Department of Pharmacology, Kagawa University, Kagawa, Japan

Correspondence: Dr N Ohashi, Internal Medicine 1, Hamamatsu University School of Medicine, 1-20-1 Handayama Higashi-ku, Hamamatsu 431-3192, Japan.

E-mail: ohashi-n@hama-med.ac.jp

Received 14 July 2015; revised 3 November 2015; accepted 3 December 2015; published online 7 January 2016 
AGT excretion could only be divided into two parts (day and nighttime) for the evaluation of circadian rhythms. Furthermore, it is unknown whether the administration of ARBs influences the circadian rhythm of intrarenal RAS components. Therefore, we conducted this study to elucidate the circadian rhythm of intrarenal RAS components and its contribution to renal damage and BP variations in rats with chronic progressive anti-thymocyte serum (ATS) nephritis. Furthermore, we attempted to clarify whether the administration of ARBs can reduce disturbances in the circadian rhythm of intrarenal RAS components, and whether its effects depend on lowering BP or blocking the RAS.

\section{METHODS}

\section{Experimental design}

All animal procedures were conducted with the approval of the Animal Committee of the Hamamatsu University School of Medicine. Six-week-old male Wistar rats weighting $150 \mathrm{~g}$ were purchased from SLC (Hamamatsu, Japan) and kept under a 12:12-h light-dark cycle (lights on at 0700 hours; Zeitgeber time (ZT) 0).

Chronic progressive ATS nephritis was induced by two consecutive injections of ATS 1 week apart after uninephrectomy, as described previously. ${ }^{11,12}$ Rats were randomly assigned into the following four groups:

Group C $(n=24)$ : rats were subjected to a sham operation on day -7 , received two injections of $0.35 \mathrm{ml}$ of $0.9 \%$ saline solution instead of ATS through the tail vein on days 0 and 7 , and were provided with standard food and water.

Group A $(n=32)$ : rats were subjected to right nephrectomy 7 days before the first ATS injection, received two injections of $0.35 \mathrm{ml}$ of ATS through the tail vein on days 0 and 7 , and were provided with standard food and drinking water.

Groups $\mathrm{AO}(n=32)$ and $\mathrm{AH}(n=32)$ : rats treated similar to those in group A received $10 \mathrm{mg} \mathrm{kg}^{-1}$ per day of olmesartan medoxomil (CS-866, Daiichi Sankyo, Tokyo, Japan) mixed with powdered food, or $5 \mathrm{mg} \mathrm{kg}^{-1}$ per day of hydralazine hydrochloride (Wako, Osaka, Japan) added to drinking water to lower the BP to equal that in $\mathrm{C}$ group, as previously described. ${ }^{12}$

Animals from all groups were euthanized by decapitation every $6 \mathrm{~h}$ from ZT 6 on day 20 to ZT 24 on day 21 . Urine samples were collected using metabolic cages for $6 \mathrm{~h}$, and BP was measured using a non-invasive tail-cuff method (BP 98A; Softron, Tokyo, Japan) before euthanasia. Blood samples and kidneys were collected immediately after decapitation and prepared as described below. It is considered that the samples at ZT 24 are equal to those at ZT 0 , because chronic model is used in our experiments, and the same diurnal variation occurs repeatedly as described previously. ${ }^{9}$

\section{Measurement of renal function and urinary protein excretion}

Serum and urinary creatinine $(\mathrm{Cr})$ concentrations were measured by enzymatic assays (Falco SD, Kyoto, Japan). Cr clearance was calculated as previously described. ${ }^{13}$ Urinary protein excretion was measured using a pyrogallol red-molybdate protein assay kit (Wako, Osaka, Japan).

\section{Evaluation of glomerular and tubulointerstitial lesions}

Kidney tissues were fixed in $4 \%$ paraformaldehyde in phosphate-buffered saline and embedded in paraffin. Tissue sections $(3 \mu \mathrm{m})$ were stained with periodic acid-Schiff and Masson's trichrome for histopathological evaluation of glomerular and tubulointerstitial lesions. To assess the glomerular changes, glomerular damage scores were evaluated using our traditional methods with minor modification. ${ }^{14}$ Briefly, a semi-quantitative grade of severity $(0,1,2$ or 3 , indicating that the feature was absent, mild, moderate or sever, respectively) was assigned to each of the following morphologic findings: (1) mononuclear cell infiltration, (2) mesangial matrix accumulation and (3) mesangial cell proliferation. Composite scores were calculated by summing individual scores in 20 glomeruli from each rat in microscopic fields observed at $\times 400$ magnification. Means of these values were then calculated. The percentages of tubulointerstitial fibrosis were evaluated in microscopic fields observed at $\times 100$ magnification. Ten microscopic fields were evaluated for each rat using a point-counting method, and mean values were calculated. All quantitative analyses were performed in a blind manner to avoid bias.

\section{Measurement of circulating RAS concentrations and urinary AGT excretion levels}

Plasma renin activity (PRA) and plasma AngII levels were measured by radioimmunoassay kits (SRL, Tokyo, Japan) and plasma AGT levels were measured by ELISA (IBL, Takasaki, Japan) to evaluate the circulating RAS. In addition, the levels of urinary AGT as a surrogate marker of intrarenal RAS activity were measured by ELISA (IBL, Takasaki, Japan) as previously described. ${ }^{15}$

\section{Immunoblot and immunohistochemical analysis of intrarenal RAS} components

To evaluate the circadian rhythm of intrarenal RAS components, immunoblot analysis of AGT, AT1R, renin, prorenin, (pro)renin receptor (PRR) and angiotensin-converting enzyme (ACE) was conducted as previously described. ${ }^{12}$ The primary antibodies were rabbit anti-AGT antibody (IBL, Takasaki, Japan), rabbit anti-AT1R antibody (Santa Cruz Biotechnology, Santa Cruz, CA, USA), rabbit anti-renin/prorenin antibody (generously provided by Dr T Senbonmatsu of Saitama Medical University, Moroyama, Japan and Dr T Inagami of Vanderbilt University, Nashville, TN, USA), rabbit anti-PRR antibody (Abcam, Tokyo, Japan) and goat anti-ACE antibody (Santa Cruz Biotechnology). Mouse anti-glyceraldehyde-3-phosphate dehydrogenase (GAPDH; Santa Cruz Biotechnology) was also used. The accumulation of these proteins as determined by immunoblot analysis was normalized against that of GAPDH.

Immunostaining for AGT, renin and prorenin, ACE, PRR and AngII in kidney sections was conducted using a Histofine kit (Nichirei-Bioscience, Tokyo, Japan) as previously described. ${ }^{11,12}$ The antibodies used for immunublot analysis were used for immunostaining (rabbit anti-AGT antibody, rabbit anti-renin/prorenin antibody, goat anti-ACE antibody and rabbit anti-PRR antibody). Rabbit anti-AngII antibody (Phoenix Pharmaceuticals, Burlingame, CA, USA) was used, and the immunoreactivity of AngII was quantitatively evaluated by a semi-automatic image analysis system using Image Pro Plus software (Media Cybernetics, Rockville, MD, USA). ${ }^{11,16}$ Twenty microscopic fields were examined for each slide, and the averaged AngII-positive areas except for vascular and glomerular lesions were obtained. Sections incubated without primary antibodies were used as a control.

\section{Immunoblot analysis of AGT in the liver}

To evaluate the circadian rhythm of AGT in the liver, immunoblot analysis of liver AGT was conducted. The primary antibody of AGT in the liver was the same one used for the kidney analysis.

\section{Statistical analysis}

Results are expressed as means \pm s.e. Significant differences among the groups were determined using analysis of variance followed by Tukey's or GamesHowell's post-hoc analysis, as appropriate. Logarithmic transformation was used for urinary AGT/Cr ratios because they were not normally distributed. ${ }^{10,17}$ $P$-value of $<0.05$ was considered statistically significant. Statistical analyses were performed using SPSS software, version 20 (SPSS, Chicago, IL, USA).

The characteristics of experimental models were determined by all the samples collected at 4 different time points. The averages of collected values for $\mathrm{BPs}$, urinary protein/Cr ratio (U-P/Cr), urinary AGT/Cr ratio (U-AGT/Cr) and circulating RAS components (PRA, plasma AGT and plasma AngII) in each time point were calculated to evaluate the amplitude of circadian oscillations. The maximum or minimum amplitude of the circadian oscillations in each group was determined as the value of peak or trough time point, respectively, and the peak-to-trough $(\mathrm{P} / \mathrm{T})$ ratio was calculated as previously described. ${ }^{9}$

\section{RESULTS}

Body weight, BP and renal damage

Body weight in group $\mathrm{C}$ was heavier than in other groups (Figure 1a). Systolic BP (SBP) and U-P/Cr in group A were significantly higher than in group $\mathrm{C}$ (Figure $1 \mathrm{~b}$ and $\mathrm{c}$ ). Cr clearance in group A were lower 
a
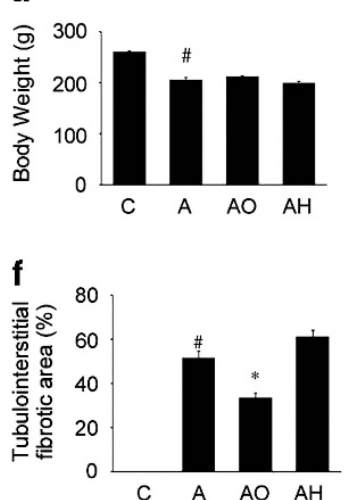

b

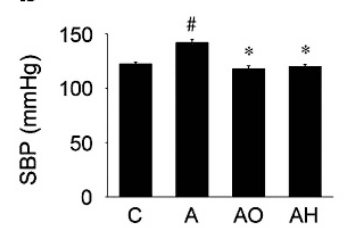

c

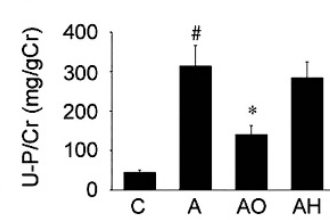

d
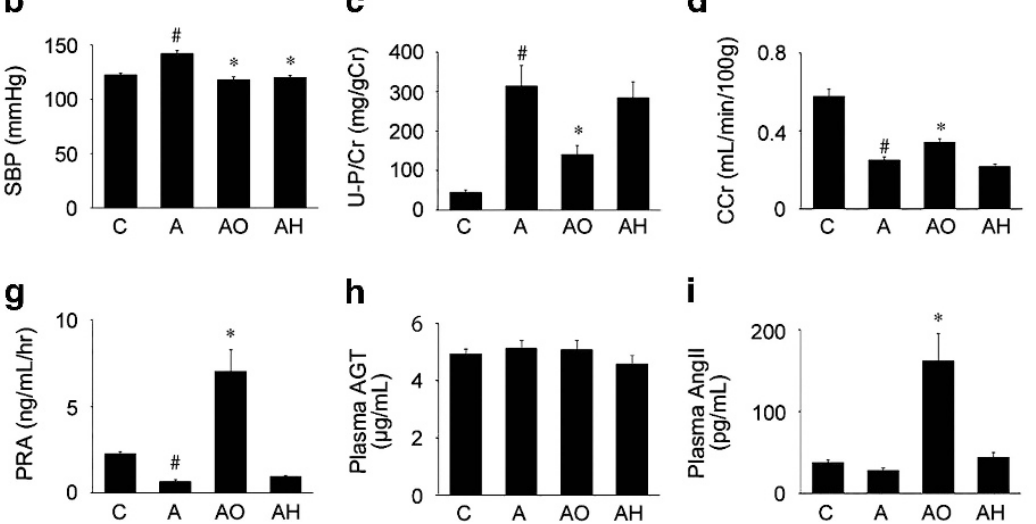

h

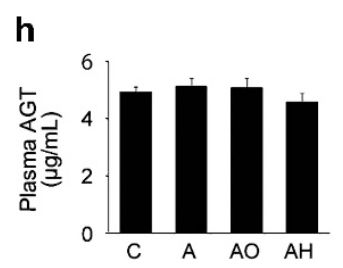

i

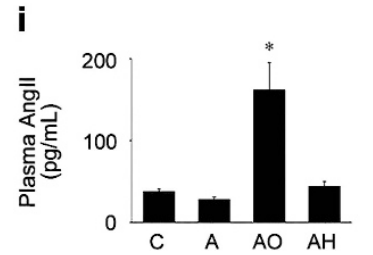

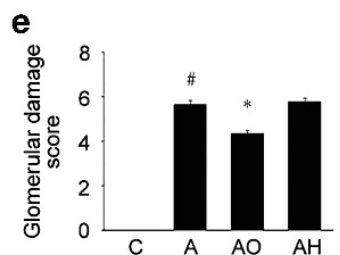

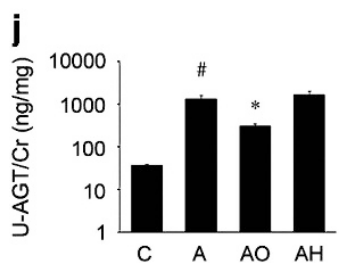

k

C
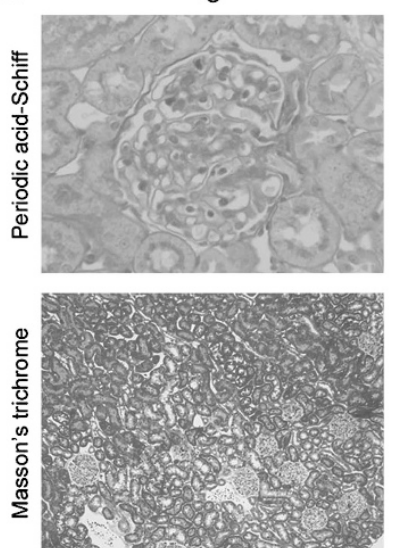
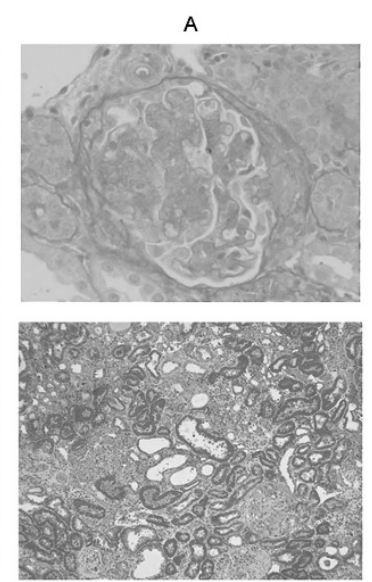

$\mathrm{AO}$
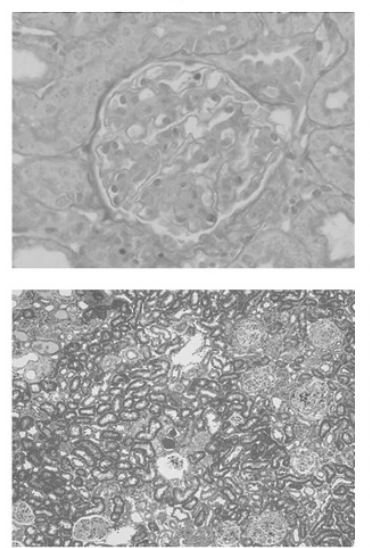

$\mathrm{AH}$

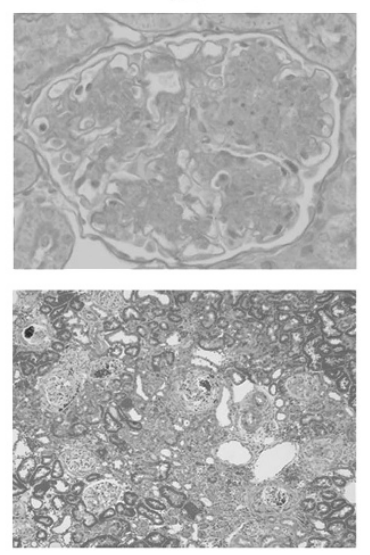

Figure 1 Values of (a) body weight, (b) systolic blood pressure (SBP), (c) urinary protein/creatinine ratio (U-P/Cr), (d) creatinine clearance (CCr), (e) glomerular damage scores: The scores are the composite ones that were calculated by summing each of the following morphologic findings: (1) mononuclear cell infiltration, (2) mesangial matrix accumulation and (3) mesangial cell proliferation. (f) Tubulointerstitial fibrotic area: The percentages of tubulointerstitial fibrosis are evaluated in 10 microscopic fields for each rat using a point-counting method. (g) Plasma renin activity (PRA), (h) plasma angiotensinogen (AGT), (i) plasma angiotensin II (AngII), (j) urinary AGT/creatinine ratio (U-AGT/Cr) at day 21 and (k) histopathological findings at day 21: Representative photomicrographs of glomeruli (original magnification $\times 400$ ) and tubulointerstitium (original magnification $\times 100$ ) for all groups. In group A, glomerular damage such as mesangiolysis, mesangial cell proliferation and mesangial matrix accumulation, and tubulointerstitial damage such as tubular atrophy and interstitial fibrosis are noted, and these finding are reduced in group $\mathrm{AO}$ but not in group $\mathrm{AH}$. These data are determined by all the samples collected at four different time points. Data represent means \pm s.e. ${ }^{\#} P<0.05$ group $\mathrm{C}$ vs. group $\mathrm{A} .{ }^{*} P<0.05$ group $\mathrm{A}$ vs. groups $\mathrm{AO}$ and $\mathrm{AH}$. A full color version of this figure is available at the Hypertension Research journal online.

than in group C (Figure 1d). Olmesartan medoxomil or hydralazine was administered to lower the $\mathrm{BP}$ in groups $\mathrm{AO}$ and $\mathrm{AH}$ to the same level as in group C (Figure 1b). SBP in active phase (ZT 18 and 24) was higher than in rest phase (ZT 6 and 12) in group C. However, although diurnal BP rhythm were not similar, SBP between active and rest phases were not different in the diseased groups (groups $\mathrm{A}, \mathrm{AO}$ and $\mathrm{AH}$; Supplementary Table 1). U-P/Cr and $\mathrm{Cr}$ clearance parameters in group $\mathrm{AO}$ were significantly improved, but those in group $\mathrm{AH}$ were not significantly different than those in group A (Figure 1c and d).

\section{Histopathological findings}

Glomerular damage, such as mononuclear cell infiltration, mesangial matrix accumulation and mesangial cell proliferation, and tubulointerstitial damage, such as tubular atrophy and interstitial fibrosis, were significantly more increased in group A than in group C. Glomerular and tubulointerstitial damage were significantly improved in group AO compared with group A. However, there were no significant differences in these types of damage between group A and group $\mathrm{AH}$ (Figure $1 \mathrm{e}, \mathrm{f}$ and $\mathrm{k}$ ).

\section{Circulating RAS and urinary AGT excretion}

In groups $\mathrm{A}$ and $\mathrm{AH}, \mathrm{PRA}$ level was decreased compared with that in group $C$, probably due to suppression by fluid retention. PRA level was significantly more increased in group $\mathrm{AO}$ than in group $\mathrm{A}$ (Figure 1g). The levels of plasma AngII in group AO were significantly higher than those in other groups, and there were no significant differences among the other groups (Figure 1i). On the other hand, the levels of plasma AGT were not significantly different among all the groups (Figure 1h). These parameters were not correlated with values for $\mathrm{U}-\mathrm{P} / \mathrm{Cr}$, glomerular and tubulointerstitial damage, renal function and BP.

In contrast, $\mathrm{U}-\mathrm{AGT} / \mathrm{Cr}$ was significantly more increased in group A than in group C. U-AGT/Cr was decreased in group AO but not in group $\mathrm{AH}$ (Figure 1j). These changes were correlated with values for $\mathrm{U}-\mathrm{P} / \mathrm{Cr}$, glomerular and tubulointerstitial damage and renal function.

Circadian rhythms of SBP, urinary protein excretion, circulating RAS components and urinary AGT excretion

Rats were awake from ZT 12 to 24 (active phase) and asleep from ZT 0 to 12 (rest phase) in order to replicate their nocturnal sleeping 
a

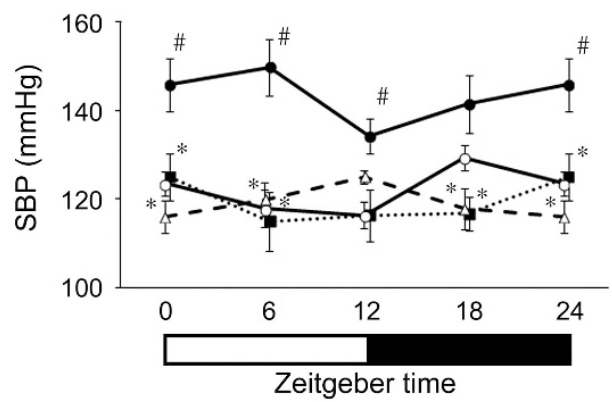

C
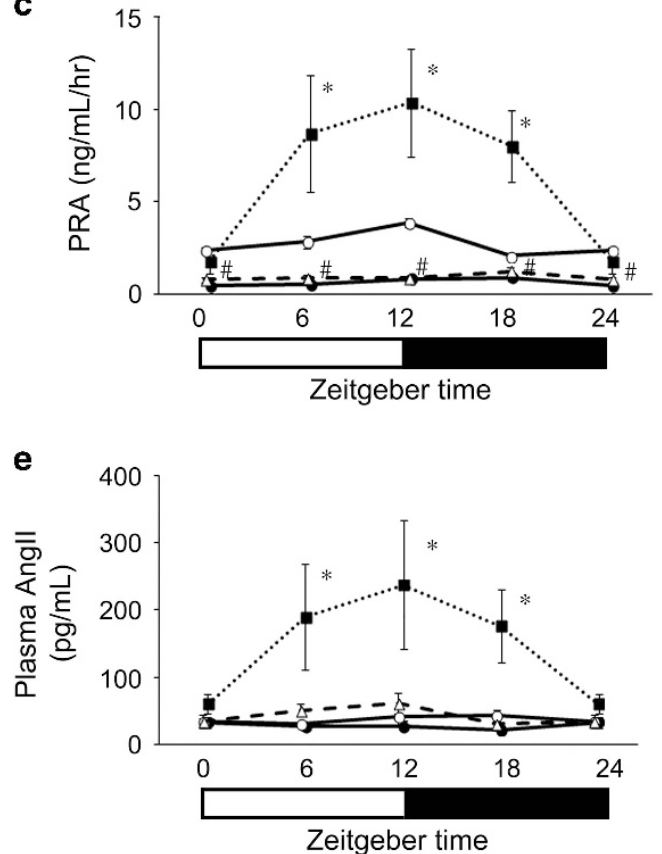

b

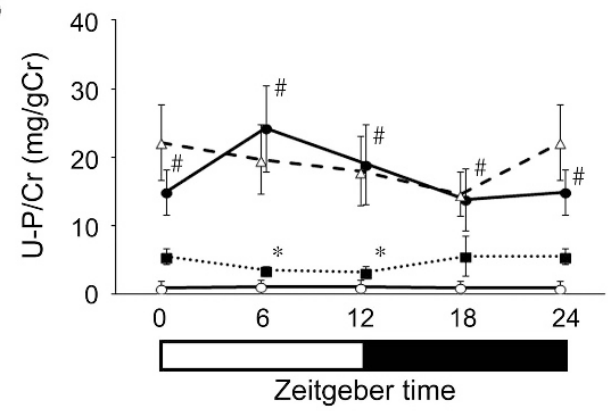

d
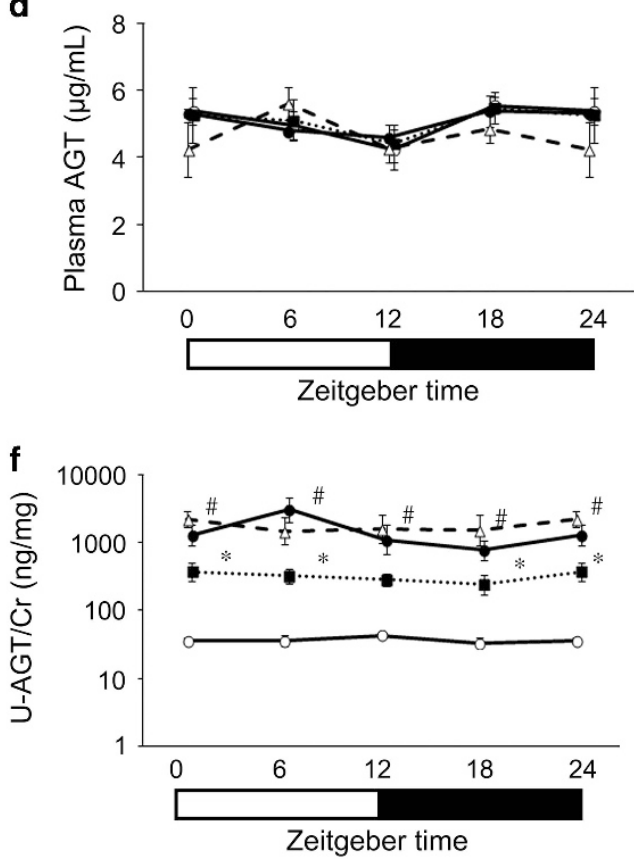

Figure 2 Circadian fluctuation of (a) systolic blood pressure (SBP), (b) urinary protein/creatinine ratio (U-P/Cr), (c) plasma renin activity (PRA), (d) plasma angiotensinogen (AGT), (e) plasma angiotensin II (AngII) and (f) urinary AGT/creatinine ratio (U-AGT/Cr) at day 21 . The solid lines with open circles show group $C$, the solid lines with closed circles show group $A$, the dotted lines with closed squares show group $A 0$ and the dashed lines with open triangles show group $\mathrm{AH}$. Data represent means \pm s.e. ${ }^{\#} P<0.05$ group $\mathrm{C}$ vs. group $\mathrm{A}$. ${ }^{\star} P<0.05$ group $\mathrm{A}$ vs. groups $\mathrm{AO}$ and $\mathrm{AH}$.

patterns. In group C, SBP peaked during the active phase (ZT 18) and tended to decrease during the rest phase. SBP in group A was higher than that in group $\mathrm{C}$ at many time points, and peaked at ZT 6. SBP in groups $\mathrm{AO}$ and $\mathrm{AH}$ was significantly lower than those in group $\mathrm{A}$, and not significantly different than those in group C except for at ZT 12 in group AH (Figure 2a). The amplitude of the oscillation of SBP evaluated by $\mathrm{P} / \mathrm{T}$ ratio in each group were not different (C: 1.11; A: 1.12; $\mathrm{AO}: 1.09$ and $\mathrm{AH}: 1.08$, respectively).

$\mathrm{U}-\mathrm{P} / \mathrm{Cr}$ in group $\mathrm{C}$ was similarly low at all time points. $\mathrm{P} / \mathrm{T}$ ratio of $\mathrm{U}-\mathrm{P} / \mathrm{Cr}$ was 1.13 in group $\mathrm{C}$. In group $\mathrm{A}, \mathrm{U}-\mathrm{P} / \mathrm{Cr}$ was significantly higher than that in group $\mathrm{C}$ at all time points. U-P/Cr peaked at ZT 6 in group $\mathrm{A}$, and the $\mathrm{P} / \mathrm{T}$ ratio of $\mathrm{U}-\mathrm{P} / \mathrm{Cr}$ was increased up to 1.73 . $\mathrm{U}-\mathrm{P} / \mathrm{Cr}$ in group $\mathrm{AO}$ but not group $\mathrm{AH}$ was low compared with that in group A at almost all time points, and fluctuations of $\mathrm{U}-\mathrm{P} / \mathrm{Cr}$ in groups $\mathrm{AO}$ and $\mathrm{AH}$ were diminished to 1.57 and 1.52 , respectively (Figure 2b).

The levels of PRA peaked late during the rest phase in group C, and those in groups A and $\mathrm{AH}$ were significantly suppressed. In group $\mathrm{AO}$, the levels of PRA exhibited large deviations and peaked during the end of the rest phase (Figure 2c). In addition, the levels of plasma AngII also exhibited large deviations and were higher in group $\mathrm{AO}$ than those in groups $\mathrm{C}$ and $\mathrm{A}$. The circadian rhythm of plasma AngII was comparable to that of PRA. There were no significant changes in this parameter among groups $\mathrm{C}, \mathrm{A}$ and $\mathrm{AH}$ (Figure 2e). On the other hand, the levels of plasma AGT did not show the obvious circadian rhythm (Figure 2d).

U-AGT/Cr levels in group A was extremely high compared than those in group $\mathrm{C}$ at all time points, and peaked at ZT 6 (the same time point as for the peak of SBP and U-P/Cr). U-AGT/Cr was significantly decreased in group $\mathrm{AO}$ at almost all time points, but less so in group $\mathrm{AH}$ compared with group A (Figure 2f). Fluctuations in U-AGT/Cr evaluated by $\mathrm{P} / \mathrm{T}$ ratio were extremely increased in group A compared with group $\mathrm{C}$, and were decreased in group $\mathrm{AO}$ and $\mathrm{AH}$ compared with group A (P/T ratio of urinary AGT in C: 1.24 ; A: 4.21 ; AO: 1.43 and $\mathrm{AH}: 1.23)$. The levels of circulating RAS components were not correlated with urinary AGT excretion levels.

\section{Circadian rhythm of protein expression for the intrarenal RAS}

Next, we evaluated whether the intrarenal RAS has a circadian rhythm. Immunoblot analysis for AGT expression in group C exhibited little 
fluctuation ( $\mathrm{P} / \mathrm{T}$ ratio 1.13). In contrast, the levels of AGT protein in group A were enhanced at all time points compared with those in group C, and peaked at ZT 6. The amplitude of oscillations of AGT protein by $\mathrm{P} / \mathrm{T}$ ratio in group A was increased up to 1.47. AGT expression was significantly low at several time points in group AO, but not in group $\mathrm{AH}$ compared with that in group A (Figure 3a and $\mathrm{b}$ ). The $\mathrm{P} / \mathrm{T}$ ratios of AGT protein in groups $\mathrm{AO}$ and $\mathrm{AH}$ were diminished to 1.24 and 1.22 , respectively. The most obvious difference in AGT protein levels among the groups was at ZT 6, and immunostaining was performed at this time to confirm the results of immunoblot analysis. Immunostaining for AGT protein in group C revealed slight expression in the proximal tubular cells, and dramatically increased the expression in group A. Although intrarenal AGT expression was reduced in group $\mathrm{AO}$, there was no significant difference between groups $\mathrm{A}$ and $\mathrm{AH}$ (Figure 3c).

Because it is not possible to detect AngII by immunoblot analysis due to its low molecule weight $(1296.49 \mathrm{Da})$, we conducted quantitative evaluation by immunostaining. Immunostaining for AngII was weak, and AngII was mainly seen in some distal tubular cells in group C. Immunostaining for AngII in group A revealed significant expression in the proximal and distal tubules, which peaked at ZT 6. Immunostaining for AngII revealed significantly more decreased levels in group $\mathrm{AO}$ than in group $\mathrm{A}$, but no significant differences were found between groups A and AH (Figure 4a and b). The amplitude of the oscillations of AngII in group A was greater than that in $\mathrm{C}$ group. The amplitude in groups $\mathrm{AO}$ and $\mathrm{AH}$ was diminished $(\mathrm{P} / \mathrm{T}$ ratio of AngII protein in C: 1.03; A: 1.26; AO: 1.14 and $\mathrm{AH}: 1.17)$.

AT1R expression was also more prominent in group A than in group $\mathrm{C}$ at many time points, and peaked at ZT 6 (Figure 5a and b). AT1R expression was significantly lower in group AO at several time points, but was different between groups $\mathrm{A}$ and $\mathrm{AH}$. The amplitude of the oscillations of AT1R in group A was greater than that in $\mathrm{C}$ group. The amplitude was diminished in groups $\mathrm{AO}$ and $\mathrm{AH}$ $(\mathrm{P} / \mathrm{T}$ ratio of $\mathrm{AT} 1 \mathrm{R}$ protein in $\mathrm{C}: 1.36 ; \mathrm{A}: 1.51 ; \mathrm{AO}: 1.37$ and $\mathrm{AH}:$ 1.26). Fluctuations in intrarenal AngII expression matched fluctuations in intrarenal AGT and AT1R expression and urinary AGT excretion.

Renin and prorenin were detected as two neighbouring bands as previously described. ${ }^{13}$ In group C, renin and prorenin expression levels were similarly low at all time points (Figure 6a and b), but expression in group A was significantly more enhanced than that in group C. Renin and prorenin expression in group AO were significantly lower at ZT 6 and ZT 18, respectively, and tended to be decreased at other time points. However, there were no differences between groups $\mathrm{A}$ and $\mathrm{AH}$. The oscillations of renin and prorenin levels were different to those of other RAS components. The amplitudes of oscillations of renin and prorenin levels were similar among groups $\mathrm{C}, \mathrm{A}$ and $\mathrm{AH}$. However, the amplitude in group $\mathrm{AO}$ was increased compared with that in the other groups $(\mathrm{P} / \mathrm{T}$ ratio of renin in $\mathrm{C}: 1.30$; $\mathrm{A}: 1.34$; $\mathrm{AO}: 1.82$ and $\mathrm{AH}: 1.47$, and $\mathrm{P} / \mathrm{T}$ ratio of prorenin in C: 1.47; A: 1.40; AO: 1.59 and AH: 1.39; Figure 6a and b). Immunostaining for total renin, including renin and prorenin at ZT 6 , were dramatically different among the groups. In group C, significant immunostaining for total renin was observed in the juxtaglomerular cells, and was faintly observed in some collecting ducts and connecting tubules. In contrast, immunostaining for total renin was observed only in the collecting ducts and connecting tubules in groups $\mathrm{A}$ and $\mathrm{AH}$. Immunostaining of juxtaglomerular cells in group $\mathrm{AO}$ was observed equally clearly in group C. However, the immunostaining levels in collecting ducts were markedly decreased compared with those in groups A and $\mathrm{AH}$ (Figure 6c). This might explain why the circadian
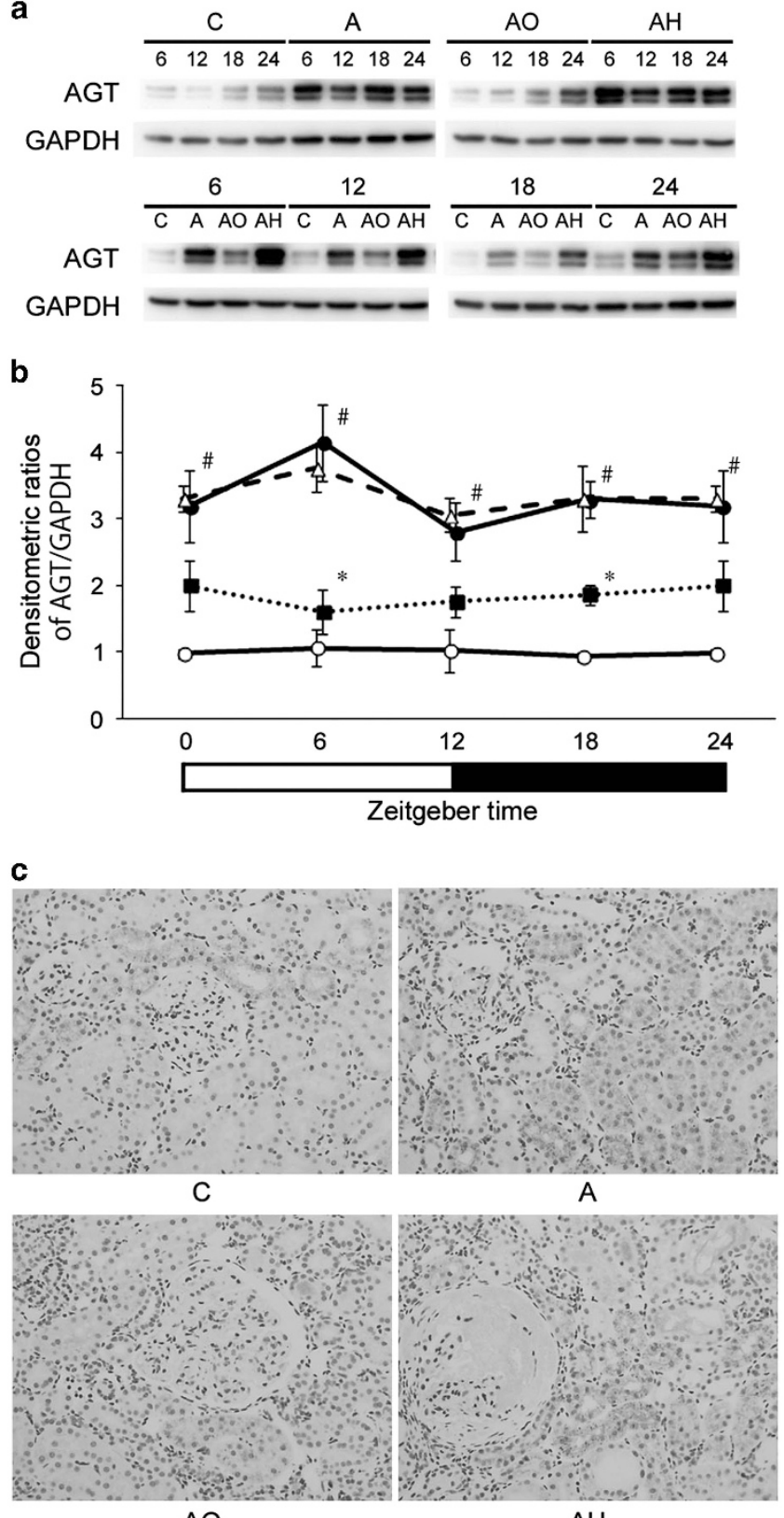

$\mathrm{AO}$

$\mathrm{AH}$

Figure 3 Immunoblot analysis for angiotensinogen (AGT) expression and its circadian fluctuation, and immunostaining for AGT at Zeitgeber time (ZT) 6. (a) Representative immunoblot data of AGT. (b) Densitometric ratios of AGT/glyceraldehyde-3-phosphate dehydrogenase (GAPDH). Densitometric ratios of AGT bands against GAPDH bands are calculated. Data represent means \pm s.e. ${ }^{\#} P<0.05$ group $C$ vs. group $A .{ }^{*} P<0.05$ group $A$ vs. group AO. The amplitude of fluctuation of AGT expression in group $A$ is greater that in group $\mathrm{C}$, and that in $\mathrm{AO}$ and $\mathrm{AH}$ groups is diminished (P/T ratio of AGT expression in $\mathrm{C}: 1.13$; $\mathrm{A}: 1.47$; $\mathrm{AO}: 1.24$ and $A H: 1.22)$. The solid line with open circles shows group $C$, the solid line with closed circles shows group A, the dotted line with closed squares shows group $A O$ and the dashed line with open triangles shows group AH. (c) Representative immunostaining for AGT at ZT 6 Immunostaining for AGT in group $C$ revealing slight expression in proximal tubular cells, and dramatically increased expression in group A. Although intrarenal AGT expression is reduced in group $A O$, that in group $A H$ is not significantly decreased compared with that in group $C$. Original magnification $\times 400$. A full color version of this figure is available at the Hypertension Research journal online. 
a

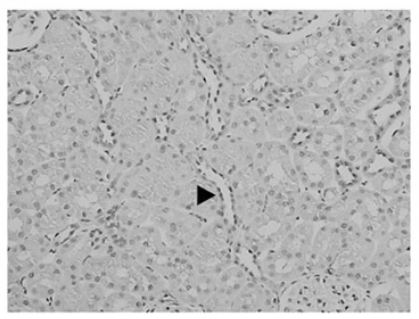

C

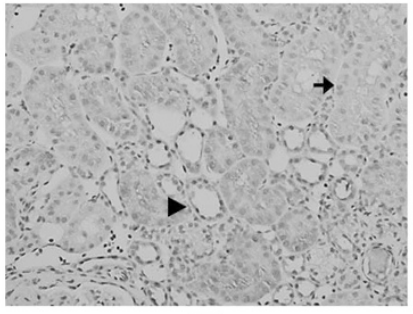

$\mathrm{AO}$

b

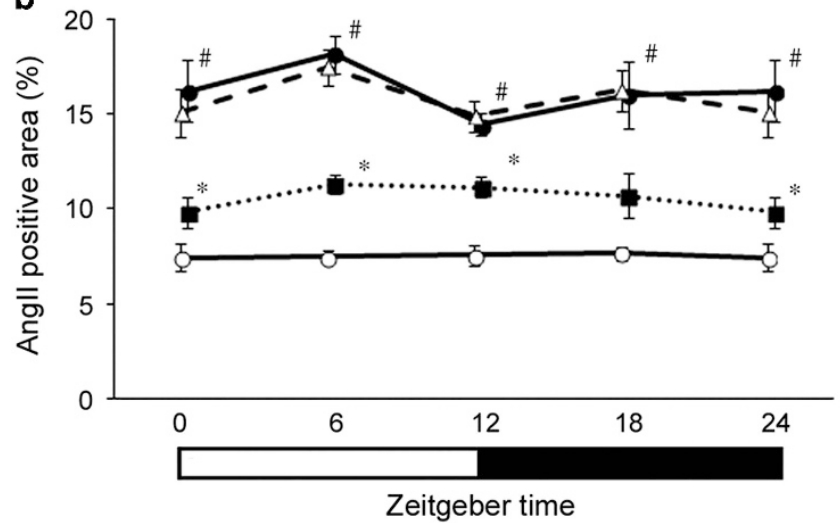

Figure 4 Immunostaining for angiotensin II (AngII) and its circadian fluctuation. (a) Representative immunostaining for Angll at Zeitgeber time (ZT) 6. Immunostaining for AngII in group $\mathrm{C}$ is weak and noted mainly in some distal tubular cells (arrowheads). Immunostaining for Angll in group A is significantly enhanced in proximal (arrows) and distal tubules (arrowheads), and that in group AO (but not in group $\mathrm{AH}$ ) is decreased compared with that in group A. Original magnification $\times 400$. (b) The degrees of immunoreactivity of Angll. The averaged immunoreactivity of Angll in 20 microscopic fields for each slide are quantitatively evaluated by a semi-automatic image analysis system using Image Pro Plus software. The amplitude of fluctuations of Angll expression in group $\mathrm{A}$ is greater that in group $\mathrm{C}$, and peaked at $\mathrm{ZT} 6$. In addition, the oscillations in $\mathrm{AO}$ and $\mathrm{AH}$ groups are diminished (P/T ratio of Angll expression in C: 1.03; $A: 1.26$; AO: 1.14 and $A H: 1.17)$. The solid line with open circles shows group $C$, the solid line with closed circles shows group $A$, the dotted line with closed squares shows group $A O$ and the dashed line with open triangles shows group $\mathrm{AH}$. Data represent means \pm s.e. ${ }^{\#} P<0.05$ group $C$ vs. group $A$. ${ }^{*} P<0.05$ group A vs. group AO. A full color version of this figure is available at the Hypertension Research journal online.

fluctuation of renin and prorenin was different to that of other RAS components.

PRR expression as determined by immunoblot analysis exhibited no remarkable changes among the different models, and the degrees of oscillation were similar among the groups (P/T ratio of PRR in C: 1.37; A: 1.24; AO: 1.28 and AH: 1.23; Supplementary Figure 1A and B). Immunostaining for PRR in the collecting ducts and connecting tubular cells at ZT 6 was observed, and the levels of immunostaining were not different among the groups (Supplementary Figure 1C). a

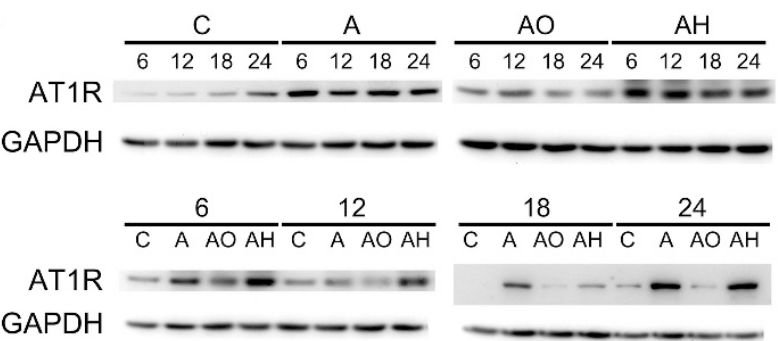

b

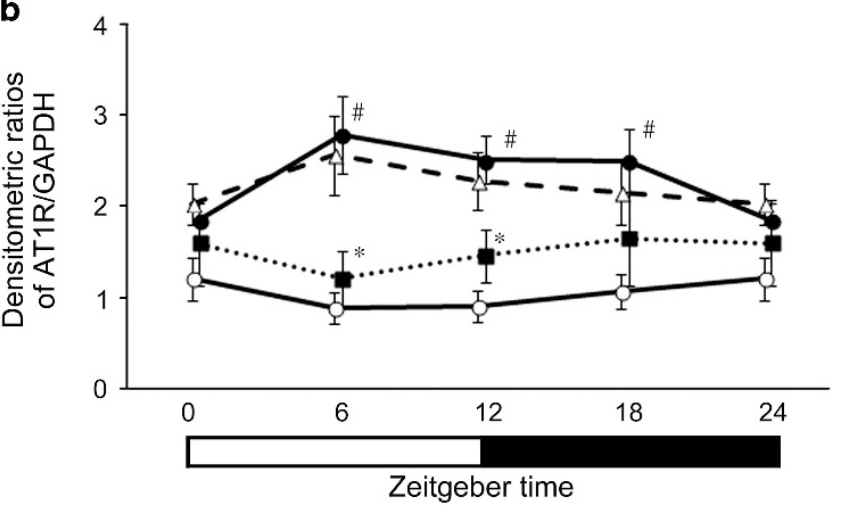

Figure 5 Immunoblot analysis for angiotensin II type 1 receptor (AT1R) and its circadian fluctuation. (a) Representative immunoblot data of AT1R. (b) Densitometric ratios of AT1R/glyceraldehyde-3-phosphate dehydrogenase (GAPDH). Densitometric ratios of AT1R bands against GAPDH bands were calculated. The amplitude of fluctuations of AT1R expression in group $A$ is greater that in group $C$, and that in $A O$ and $A H$ group is diminished $(\mathrm{P} / \mathrm{T}$ ratio of $\mathrm{AT1R}$ protein expression in $\mathrm{C}: 1.36 ; \mathrm{A}: 1.51 ; \mathrm{AO}: 1.37$ and $\mathrm{AH}$ : 1.26). The solid line with open circles shows group $C$, the solid line with closed circles shows group $\mathrm{A}$, the dotted line with closed squares shows group $\mathrm{AO}$ and the dashed line with open triangles shows group $\mathrm{AH}$. Data represent means \pm s.e. ${ }^{\#} P<0.05$ group $C$ vs. group $A .{ }^{*} P<0.05$ group $A$ vs. group $\mathrm{AO}$.

ACE expression as determined by immunoblot analysis also exhibited no significant changes among the models, and the degrees of oscillation were similar between the groups (P/T ratio of ACE in C: 1.16; A: 1.19; AO: 1.28 and AH: 1.21; Supplementary Figure 2A and B). ACE was immunostained at ZT 6 in the brush border of proximal tubules and in the endothelium of blood vessels. Immunostaining levels for ACE in both lesions were not different among the groups (Supplementary Figure 2C).

\section{Circadian rhythm of protein expression for the liver AGT}

To determine whether activation of the intrarenal RAS was influenced by AGT that was produced in the liver and filtered through glomerular basement membrane, we investigated the AGT protein expression for the liver. There were no obvious changes of expression levels and the degrees of oscillation among the groups (P/T ratio of liver AGT in C: 1.20; A: 1.19; AO: 1.23 and AH: 1.10; Supplementary Figure $3 \mathrm{~A}$ and $\mathrm{B})$.

\section{DISCUSSION}

Consecutive injections of ATS after uninephrectomy in rats caused chronic progressive renal dysfunction, augmentation of urinary protein excretion, BP elevation, glomerular and tubulointerstitial damage and activation of the intrarenal RAS independent of the circulating RAS, as previously described. ${ }^{11,12}$ In this model, intrarenal AGT, AngII and AT1R were activated and oscillated more than in 
controls. Moreover, they peaked at ZT 6, which was the same as for $\mathrm{BP}$ and urinary protein excretion. In addition, the administration of both olmesartan medoxomil and hydralazine effectively reduced the amplitude of oscillations of AGT, AngII and AT1R. However, the levels of AGT, AngII and AT1R expression in group AO but not group AH were significantly decreased compared with those in group A.

We previously reported that levels of urinary AGT excretion fluctuated only in CKD patients, and that changes of urinary AGT excretion levels in CKD patients (but not in individuals without CKD)

a
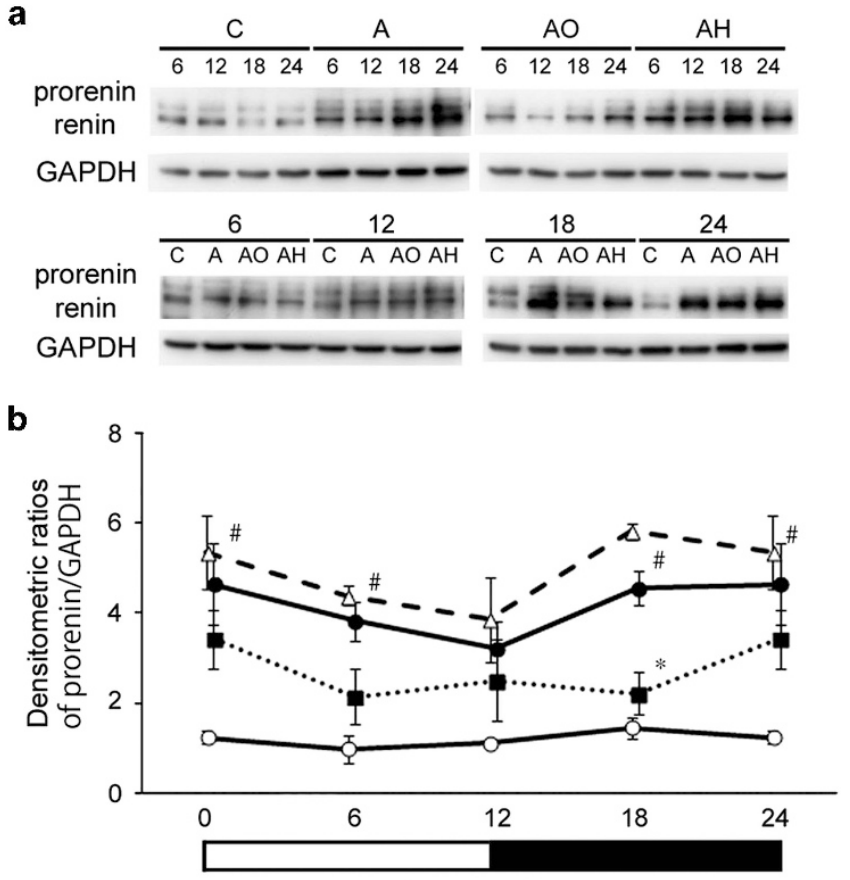

Zeitgeber time

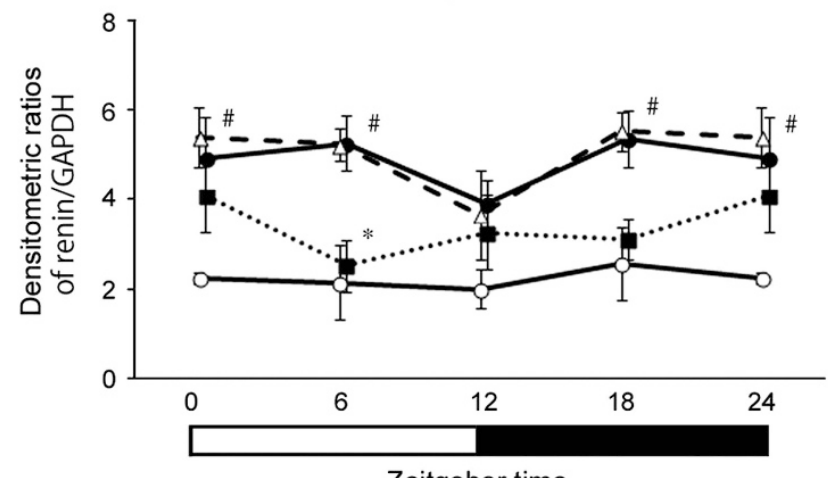

Zeitgeber time

c
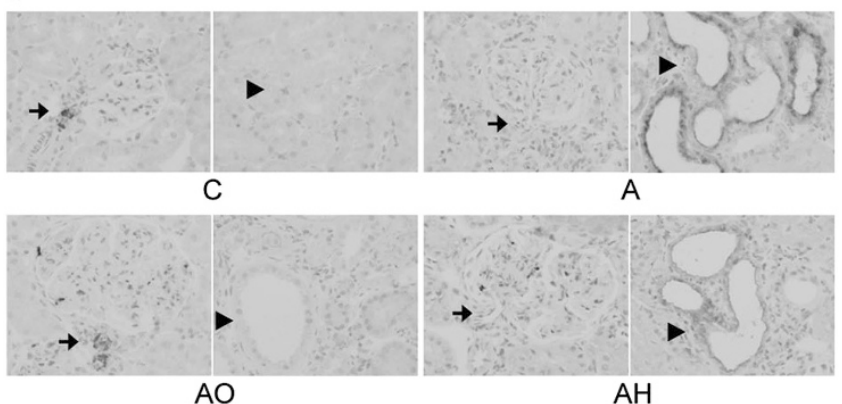

matched changes in BP and urinary protein excretion. ${ }^{10}$ In the present study, we confirmed that the peak of intrarenal RAS activation occurred at the same time as the peak of urinary protein excretion and BP elevation. We discovered augmented expression of intrarenal AGT, AngII and AT1R proteins as well as urinary AGT excretion that is a surrogate marker of intrarenal RAS was existed, and indicating the existence of fluctuations in intrarenal RAS components in this disease model.

Circadian fluctuations in intrarenal renin, AGT and AngII were clearly not related to those of circulating renin, AGT and AngII. It has long been known that the levels of circulating renin and AngII in rats have a circadian rhythm, peak late in the rest phase to early in the active phase, ${ }^{1}$ and the circulating AGT did not have a circadian rhythm. ${ }^{18,19}$ However, fluctuations in intrarenal renin levels were not the same as those of intrarenal AngII. Both intrarenal AngII and AGT exhibited the same oscillations. These results may indicate that the rate-limiting factors of the circadian rhythms of the circulating and intrarenal RAS are different. It is likely that renin is the rate-limiting factor of the circulating RAS, and that AGT is the rate-limiting factor of the intrarenal RAS.

It has been recognized that intrarenal RAS activation causes hypertension. ${ }^{4,5}$ Conversely, the intrarenal RAS is also activated by hypertension. ${ }^{20,21}$ Several studies have indicated that urinary protein excretion increases during BP elevation, ${ }^{10,22,23}$ and that increased urinary protein excretion leads to enhanced intrarenal RAS activity. ${ }^{24}$ Although AGT is generated in the proximal tubules, ${ }^{2,25}$ Matsusaka et al. demonstrated that AGT produced in the liver and filtered through the glomerular basement membrane is the primary source of intrarenal AngII. ${ }^{26}$ In our experiments, there was no circadian rhythm of liver AGT, and no significant changes of circadian rhythm were found in plasma AGT as described previously. ${ }^{18,19}$ However, it is possible that glomerular permeability that is augmented in the setting of glomerular injury and glomerular hypertension increases AGT excretion levels into the tubular lumen ${ }^{27-29}$ and circadian fluctuation of the glomerular permeability may influence that of intrarenal AGT. Once the AGT is excreted into the tubular lumen, AngII is generated because all the components to synthesize AngII exist in the tubules. $2,4,30$ This may explain why circadian fluctuations of the intrarenal RAS exist, why BP elevation, urinary protein excretion

Figure 6 Immunoblot analysis for renin and prorenin expression and their circadian fluctuations, and immunostaining for renin and prorenin at Zeitgeber time (ZT) 6. (a) Representative immunoblot data of renin and prorenin. (b) Densitometric ratios of renin and prorenin/glyceraldehyde-3phosphate dehydrogenase (GAPDH). Densitometric ratios of renin and prorenin bands against GAPDH bands are calculated. The amplitude of fluctuations of renin and prorenin expression in group $\mathrm{AO}$ is greater than that in the other groups (P/T ratio of renin in $\mathrm{C}: 1.30 ; \mathrm{A}: 1.34 ; \mathrm{AO}: 1.82$ and $\mathrm{AH}: 1.47$, and $\mathrm{P} / \mathrm{T}$ ratio of prorenin in $\mathrm{C}: 1.47 ; \mathrm{A}: 1.40 ; \mathrm{AO}$ : 1.59 and $\mathrm{AH}$ : 1.39). The solid lines with open circles show group $C$, the solid lines with closed circles show group $A$, the dotted lines with closed squares show group $\mathrm{AO}$, and the dashed lines with open triangles show group $\mathrm{AH}$. Data represent means \pm s.e. ${ }^{\#} P<0.05$ group $C$ vs. group $A .{ }^{*} P<0.05$ group $A$ vs. group AO. (c) Representative immunostaining for total renin at ZT 6 In group $C$, significant immunostaining for total renin is observed in the juxtaglomerular cells (arrows) and is faintly observed in some collecting ducts and connecting tubules (arrowheads). In contrast, immunostaining for total renin is observed only in the collecting ducts and connecting tubules in groups $\mathrm{A}$ and $\mathrm{AH}$. Immunostaining of juxtaglomerular cells in group $\mathrm{AO}$ is observed as clearly as that in group C. However, the immunostaining levels in collecting ducts are markedly decreased compared with those in groups $\mathrm{A}$ and $\mathrm{AH}$. Original magnification $\times 400$. A full color version of this figure is available at the Hypertension Research journal online. 
and intrarenal RAS activation occur in parallel in this disease model, and why fluctuations in the intrarenal RAS are not related to those in the circulating RAS.

The regulation of intrarenal AT1R is complex, because AT1R expressed in vessels, glomeruli and tubules is controlled in different manners. ${ }^{4}$ In rats fed on a low-sodium diet, AT1R expression was increased in tubules but decreased in glomeruli. ${ }^{31}$ Vascular and glomerular AT1R expression were decreased, but proximal tubular AT1R expression was sustained in AngII-infused hypertensive models. ${ }^{32}$ However, Cao et al. demonstrated that AT1R protein levels in the entire kidney were upregulated in 5/6 nephrectomized rat, and that the administration of ARBs reduced expression. ${ }^{33}$ As no good commercial antibodies are available, we could not determine the location of AT1R expression by immunostaining. However, simultaneously increased AT1R and AngII expression might aggravate the circadian rhythm of BP.

Naito et al. demonstrated that the amplitude of oscillations of the circadian mRNA expression of cardiac RAS components was increased in spontaneously hypertensive rats compared with control rats using the P/T ratio. They also showed that the amplitude of oscillations was decreased in spontaneously hypertensive rats treated with ARBs using the $\mathrm{P} / \mathrm{T}$ ratio. ${ }^{9}$ Therefore, we evaluated the circadian rhythm using $\mathrm{P} / \mathrm{T}$ ratio in our experiment. As a result, we could demonstrate that renal damage was much better in group $\mathrm{AO}$ but not in group $\mathrm{AH}$ than in group A, and that although the levels of intrarenal AGT, AngII and AT1R expression were significantly lower in group AO but not in group $\mathrm{AH}$ compared with those in group $\mathrm{A}$, the amplitudes of oscillations of these proteins were similarly diminished in groups AO and $\mathrm{AH}$ compared with those in group $\mathrm{A}$. These results indicate the intervention to the intrarenal RAS fluctuation did not improve renal damage in this model. However, the conclusion seems to be different from the previous report, which indicates that disturbed circadian rhythm of the intrarenal RAS aggravates diurnal BP rhythm and renal damage. ${ }^{10}$ One of the reasons is that the circadian rhythm of the intrarenal RAS was evaluated by the only $\mathrm{P} / \mathrm{T}$ ratio. It is possible that the same levels of $\mathrm{P} / \mathrm{T}$ ratio of the intrarenal RAS do not always show the same pattern of the circadian rhythm of the intrarenal RAS. Therefore, more careful examination is required to clarify whether the circadian rhythm of intrarenal RAS contributes to renal damage in the future.

In our study, renal function and glomerular and tubulointerstitial damage were independent of BP and the amplitude of oscillations of $\mathrm{BP}$ and intrarenal RAS protein expression levels, and dependent on the degree of intrarenal RAS protein expression. The failure of antihypertensive therapy independent of RAS blockers to prevent renal damage was consistent with the previous basic and clinical studies. ${ }^{11,34,35}$ SBPs in groups $\mathrm{AO}$ and $\mathrm{AH}$ were not statistically different in all four time points (ZT 6 to ZT 24), and SBPs between active and rest phases in each group were not different. However, diurnal $\mathrm{BP}$ rhythm in groups $\mathrm{AO}$ and $\mathrm{AH}$ was not completely similar. In addition, more short-time oscillation of BP was not evaluated. In patients with hypertension, administration of ARB increases dipper pattern of BP rhythm. ${ }^{36}$ Furthermore, Griffin et al demonstrated the differences of intraglomeular BPs in 5/6 nephrectomised rat treated with ACE inhibitor and calcium channel blocker and that glomerular damage was ameliorated by ACE inhibitor but not calcium channel blocker because of the prevention of greater pressure transmission to the glomeruli. ${ }^{37}$ Therefore, we could not completely exclude whether the differences of more short-time BP fluctuation and intraglomerular $\mathrm{BP}$ between groups $\mathrm{AO}$ and $\mathrm{AH}$ influenced the differences of renoprotective effects.
In conclusion, intrarenal AGT, AngII and AT1R levels were increased in renal tissues at the same time points. The levels of these proteins only slightly fluctuated in control rats. On the other hand, the amplitude of oscillations of these proteins was augmented in ATS nephritis rats. The administration of olmesartan medoxomil and hydralazine reduced BP and circadian fluctuations of intrarenal AGT, AngII and AT1R levels. Renal dysfunction and glomerular and tubulointerstitial damage were caused by the activation of the intrarenal RAS independent of the amplitude of its oscillations and BP.

\section{CONFLICT OF INTEREST}

The authors declare no conflict of interest.

\section{ACKNOWLEDGEMENTS}

We acknowledge Dr T Senbonmatsu (Saitama Medical University, Moroyama, Japan) and Dr T Inagami (Vanderbilt University, Nashville, TN, USA) for providing the rabbit anti-renin antibody, and Daiichi Sankyo Co. (Tokyo, Japan) for providing olmesartan medoxomil (CS-866). This study was supported by grants from the Young Investigator Research Projects of Hamamatsu University School of Medicine in 2011, 2012 and 2013 (Awarded to Naro OHASHI), and in 2012 and 2013 (Awarded to Shinsuke ISOBE).

1 Hilfenhaus M. Circadian rhythm of the renin-angiotensin-aldosterone system in the rat. Arch Toxicol 1976; 36: 305-316.

2 Kobori H, Nangaku M, Navar LG, Nishiyama A. The intrarenal renin-angiotensin system: from physiology to the pathobiology of hypertension and kidney disease. Pharmacol Rev 2007; 59: 251-287.

3 Richards AM, Nicholls MG, Espiner EA, Ikram H, Cullens M, Hinton D. Diurnal patterns of blood pressure, heart rate and vasoactive hormones in normal man. Clin Exp Hypertens A 1986; 8: 153-166.

4 Navar LG, Kobori H, Prieto MC, Gonzalez-Villalobos RA. Intratubular renin-angiotensin system in hypertension. Hypertension 2011; 57: 355-362.

5 Coffman TM, Crowley SD. Kidney in hypertension: guyton redux. Hypertension 2008; 51: 811-816.

6 Fukuda M, Urushihara M, Wakamatsu T, Oikawa T, Kobori H. Proximal tubular angiotensinogen in renal biopsy suggests nondipper BP rhythm accompanied by enhanced tubular sodium reabsorption. J Hypertens 2012; 30: 1453-1459.

7 Fukuda M, Wakamatsu-Yamanaka T, Mizuno M, Miura T, Tomonari T, Kato Y, Ichikawa T, Miyagi S, Shirasawa Y, Ito A, Yoshida A, Kimura G. Angiotensin receptor blockers shift the circadian rhythm of blood pressure by suppressing tubular sodium reabsorption. Am J Physiol Renal Physiol 2011; 301: F953-F957.

8 Kobori H, Alper AB Jr, Shenava R, Katsurada A, Saito T, Ohashi N, Urushihara M, Miyata K, Satou R, Hamm LL, Navar LG. Urinary angiotensinogen as a novel biomarker of the intrarenal renin-angiotensin system status in hypertensive patients. Hypertension 2009; 53: 344-350.

9 Naito Y, Tsujino T, Fujioka Y, Ohyanagi M, Iwasaki T. Augmented diurnal variations of the cardiac renin-angiotensin system in hypertensive rats. Hypertension 2002; 40: 827-833.

10 Isobe S, Ohashi N, Fujikura T, Tsuji T, Sakao Y, Yasuda H, Kato A, Miyajima H, Fujigaki $Y$. Disturbed circadian rhythm of the intrarenal renin-angiotensin system: relevant to nocturnal hypertension and renal damage. Clin Exp Nephrol 2015; 19: 231-239.

11 Ohashi N, Yamamoto T, Huang Y, Misaki T, Fukasawa H, Suzuki H, Togawa A, Suzuk S, Fujigaki Y, Nakagawa T, Nakamura Y, Suzuki F, Kitagawa M, Hishida A. Intrarenal RAS activity and urinary angiotensinogen excretion in anti-thymocyte serum nephritis rats. Am J Physiol Renal Physiol 2008; 295: F1512-F1518.

12 Huang $Y$, Yamamoto T, Misaki T, Suzuki H, Togawa A, Ohashi N, Fukasawa $H$, Fujigaki Y, Ichihara A, Nishiyama A, Senbonmatsu T, Ikegaya N, Hishida A. Enhanced intrarenal receptor-mediated prorenin activation in chronic progression anti-thymocyte serum nephritis rats on high salt intake. Am J Physiol Renal Physiol 2012; 303: F130-F138.

13 Hosgood SA, Mohamed IH, Nicholson ML. The two layer method does not improve the preservation of porcine kidneys. Med Sci Monit 2011; 17: BR27-BR33.

14 Fukasawa H, Yamamoto T, Suzuki H, Togawa A, Ohashi N, Fujigaki Y, Uchida C, Aoki M, Hosono M, Kitagawa M, Hishida A. Treatment with anti-TGF- $\beta$ antibody ameliorates chronic progressive nephritis by inhibiting smad/TGF- $\beta$ signalling. Kidney Int 2004; 65: 63-74.

15 Kobori H, Katsurada A, Miyata K, Ohashi N, Satou R, Saito T, Hagiwara Y, Miyashita K, Navar LG. Determination of plasma and urinary angiotensinogen levels in rodents by newly developed ELISA. Am J Phisiol Renal Physiol 2008; 294: F1257-F1263.

16 Kobori H, Ozawa Y, Suzuki Y, Nishiyama A. Enhanced intrarenal angiotensinogen contributes to early renal injury in spontaneously hypertensive rats. J Am Soc Nephrol 2005; 16: 2073-2080. 
17 Mills KT, Kobori H, Hamm LL, Alper AB, Khan IE, Rahman M, Navar LG, Liu Y, Browne GM, Batuman V, He J, Chen J. Increased urinary excretion of angiotensinogen is associated with risk of chronic kidney disease. Nephrol Dial Transplant 2012; 27: 3176-3181.

18 Lommer D, Distler A, Nast HP, Sinterhauf K, Walter U, Wolff HP, Sieler K. Diurnal profiles of plasma aldosterone, cortisol, renin, angiotensinogen and angiotensinases in normal subjects. Klin Wochenschr 1976; 54: 123-130.

19 Nishijima Y, Kobori H, Kaifu K, Mizushige T, Hara T, Nishiyama A, Kohno M. Circadian rhythm of plasma and urinary angiotensinogen in healthy volunteers and in patients with chronic kidney disease. J Renin Angiotensin Aldosterone Syst 2014; 15: 505-508.

20 Graciano ML, Cavaglieri Rde C, Dellé H, Dominguez WV, Casarini DE, Malheiros DM, Noronha IL. Intrarenal renin-angiotensin system is upregulated in experimental model of progressive renal disease induced by chronic inhibition of nitric oxide synthesis. J Am Soc Nephrol 2004; 15: 1805-1815.

21 Gonzalez-Villalobos RA, Janjoulia T, Fletcher NK, Giani JF, Nguyen MT, Riquier-Brison AD, Seth DM, Fuchs S, Eladari D, Picard N, Bachmann S, Delpire E, Peti-Peterdi J, Navar LG, Bernstein KE, McDouough AA. The absence of intrarenal ACE protects against hypertension. J Clin Invest 2013; 123: 2011-2023.

22 Fukuda M, Munemura M, Usami T, Nakao N, Takeushi O, Kamiya Y, Yoshida A, Kimura G. Nocturnal blood pressure is elevated with natriuresis and proteinuria as renal function deteriorates in nephropathy. Kidney Int 2004; 65: 621-625.

23 Nishimura M, Uzu T, Fujii T, Kimura G. Disturbed circadian rhythm of urinary albumin excretion in non-dipper type of essential hypertension. Am J Nephrol 2002; 22: 455-462.

24 Kobori H, Ohashi N, Katsurada A, Miyata K, Satou R, Saito T, Yamamoto T. Urinary angiotensinogen as a potential biomarker of severity of chronic renal disease. J Am Soc Hypertens 2008; 2: 349-354.

25 Kobori H, Nishiyama A, Harrison-Bernard LM, Navar LG. Urinary angiotensinogen as an indicator of intrarenal angiotensin status in hypertension. Hypertension 2003; 41: 42-49.

26 Matsusaka T, Niimura F, Shimizu A, Pastan I, Saito A, Kobori H, Nishiyama A, Ichikawa I. Liver angiotensinogen is the primary source of renal angiotensin II. J Am Soc Nephrol 2012; 23: 1181-1189.
27 Anderson S, Meyer TW, Rennke HG, Brenner BM. Control of glomerular hypertension limits glomerular injury in rats with reduced renal mass. J Clin Invest 1985; 76: 612-619.

28 Olson JL, Hostetter TH, Rennke HG, Brenner BM, Venkatachalam MA. Altered glomerular permselectivity and progression sclerosis following extreme ablation of renal mass. Kidney Int 1982; 22: 112-126.

29 Matsusaka T, Niimura F, Pastan I, Shintani A, Nishiyama A, Ichikawa I. Podocyte injury enhances filtration of liver-derived angiotensinogen and renal angiotensin II generation. Kidney Int 2014; 85: 1068-1077.

30 Navar LG, Prieto MC, Satou R, Kobori H. Intrarenal angiotensin II and its contribution to the genesis of chronic hypertension. Curr Opin Pharmacol 2011; 11: 180-186.

31 Cheng HF, Becker BN, Burns KD, Harris RC. Angiotensin II upregulates type-1 angiotensin II receptors in renal proximal tubule. J Clin Invest 1995; 95: 2012-2019.

32 Harrison-Bernard LM, Zhuo J, Kobori H, Ohishi M, Navar LG. Intrarenal AT(1) receptor and ACE binding in ANG II-induced hypertensive rats. Am J Physiol Renal Physiol 2002; 282: F19-F25.

33 Cao W, Li A, Wang L, Zhou Z, Su Z, Bin W, Wilcox CS, Hou FF. A salt-induced renocerebral reflex activates renin-angiotensin systems and promotes CKD progression. J Am Soc Nephrol 2015; 26: 1619-1633.

34 Wright JT Jr, Bakris G, Greene T, Agodoa LY, Appel LJ, Cheek D, Douglas-Baltimore JG, Gassman J, Glassock R, Hebert L, Jamerson K, Lewis J, Phillips RA, Toto RD, Middleton JP, Rostand SG. Effect of blood pressure lowering and antihypertensive drug class on progression of hypertensive kidney disease: results from the AASK trial. JAMA 2002; 288: 2421-2431.

35 Hošková L, Málek I, Kautzner J, Honsová E, van Dokkum RP, Husková Z, Vojtišková A, Varcabová S, Cervenka L, Kopkan L. Tacrolimus-induced hypertension and nephrotoxicity in Fawn-Hooded rats are attenuated by dual inhibition of renin-angiotensin system. Hypertens Res 2014; 37: 724-732.

36 Gosse $\mathrm{P}$, Schumacher $\mathrm{H}$. Effect of telmisartan vs ramipril on 'dipping' status and blood pressure variability: pooled analysis of the PRISMA studies. Hypertens Res 2014; 37: $151-157$.

37 Griffin KA, Picken MM, Bidani AK. Deleterious effects of calcium channel blocker on pressure transmission and gromerular injury in rat remnant kidneys. J Clin Invest 1995; 96: $793-800$

Supplementary Information accompanies the paper on Hypertension Research website (http://www.nature.com/hr) 